\title{
Automatic Detection of Poor Speech Recognition at the Dialogue Level
}

\author{
Diane J. Litman, Marilyn A. Walker and Michael S. Kearns \\ AT\&T Labs Research \\ 180 Park Ave, Bldg 103 \\ Florham Park, N.J. 07932 \\ \{diane, walker, mkearns\}@research.att.com
}

\begin{abstract}
The dialogue strategies used by a spoken dialogue system strongly influence performance and user satisfaction. An ideal system would not use a single fixed strategy, but would adapt to the circumstances at hand. To do so, a system must be able to identify dialogue properties that suggest adaptation. This paper focuses on identifying situations where the speech recognizer is performing poorly. We adopt a machine learning approach to learn rules from a dialogue corpus for identifying these situations. Our results show a significant improvement over the baseline and illustrate that both lower-level acoustic features and higher-level dialogue features can affect the performance of the learning algorithm.
\end{abstract}

\section{Introduction}

Builders of spoken dialogue systems face a number of fundamental design choices that strongly influence both performance and user satisfaction. Examples include choices between user, system, or mixed initiative, and between explicit and implicit confirmation of user commands. An ideal system wouldn't make such choices a priori, but rather would adapt to the circumstances at hand. For instance, a system detecting that a user is repeatedly uncertain about what to say might move from user to system initiative, and a system detecting that speech recognition performance is poor might switch to a dialogue strategy with more explicit prompting, an explicit confirmation mode, or keyboard input mode. Any of these adaptations might have been appropriate in dialogue D1 from the Annie system (Kamm et al., 1998), shown in Figure 1.

In order to improve performance through such adaptation, a system must first be able to identify, in real time, salient properties of an ongoing dialogue that call for some useful change in system strategy. In other words, adaptive systems should try to automatically identify actionable properties of ongoing dialogues.

Previous work has shown that speech recognition performance is an important predictor of user satisfaction, and that changes in dialogue behavior impact speech recognition performance (Walker et al., 1998b; Litman et al., 1998; Kamm et al., 1998). Therefore, in this work, we focus on the task of automatically detecting poor speech recognition performance in several spoken dialogue systems developed at AT\&T Labs. Rather than hand-crafting rules that classify speech recognition performance in an ongoing dialogue, we take a machine learning approach. We begin with a collection of system logs from actual dialogues that were labeled by humans as having had "good" or "bad" speech recognition (the training set). We then apply standard machine learning algorithms to this training set in the hope of discovering, in a principled manner, classifiers that can automatically detect poor speech recognition during novel dialogues.

In order to train such classifiers, we must provide them with a number of "features" of dialogues derived from the system logs that might allow the system to automatically identify poor recognition performance. In addition to identifying features that provide the best quantitative solutions, we are also interested in comparing the performance of classifiers derived solely from acoustic features or from "high-level" dialogue features, and from combinations of these and other feature types. Note that we are free to invent as many features as we like, as long as they can be computed in real time from the raw system logs.

Since the dialogue systems we examine use automatic speech recognition (ASR), one obvious feature available in the system $\log$ is a per-utterance score from the speech recognizer representing its "confidence" in its interpretation of the user's utterance (Zeljkovic, 1996). For dialogue D1, the recognizer's output and the associated confidence scores 


\begin{tabular}{|c|c|c|c|}
\hline A1: & $\begin{array}{l}\text { Actual Dialogue } \\
\text { Annie here. }\end{array}$ & ASR Interpretation & ASR Confidence \\
\hline U1: & Hello Annie, could you give me the information on a meeting I have tomorrow? & REJECT & -4.84 \\
\hline A2: & What was that? & & \\
\hline $\begin{array}{l}\text { U2: } \\
\text { A3: }\end{array}$ & $\begin{array}{l}\text { I need to find out uh, a meeting time and place. Kim sent me a message on it. } \\
\text { For all options say help me out. }\end{array}$ & REJECT & -4.20 \\
\hline U3: & Help me out. & REJECT & -6.35 \\
\hline A4: & Annie here. & & \\
\hline U4: & Help. & help & -1.74 \\
\hline A5: & $\begin{array}{l}\text { Help. Say call or say send a message, followed by a label or a number. } \\
\text { Or say get my messages... }\end{array}$ & & \\
\hline U5: & Get messages. (user interrupts A5) & get me sips & -3.33 \\
\hline A6: & Speech and Image Processing Lab. & & \\
\hline U6: & Help. & help & -1.78 \\
\hline A7: & Help. Say the first and last name ... & & \\
\hline U7: & Kim. & annie & -3.81 \\
\hline
\end{tabular}

Figure 1: Example dialogue excerpt D1 with Annie.

are in the last two columns of Figure 1. These confidence measures are based on the recognizer's language and acoustic models. The confidence scores are typically used by the system to decide whether it believes it has correctly understood the user's utterance. When the confidence score falls below a threshold defined for each system, the utterance is considered a rejection (e.g., utterances $\mathrm{U} 1, \mathrm{U} 2$, and $\mathrm{U} 3$ in D1). Note that since our classification problem is defined by speech recognition performance, it might be argued that this confidence feature (or features derived from it) suffices for accurate classification.

However, an examination of the transcript in D1 suggests that other useful features might be derived from global or high-level properties of the dialogue history, such as features representing the system's repeated use of diagnostic error messages (utterances A2 and A3), or the user's repeated requests for help (utterances U4 and U6).

Although the work presented here focuses exclusively on the problem of automatically detecting poor speech recognition, a solution to this problem clearly suggests system reaction, such as the strategy changes mentioned above. In this paper, we report on our initial experiments, with particular attention paid to the problem definition and methodology, the best performance we obtain via a machine learning approach, and the performance differences between classifiers based on acoustic and higherlevel dialogue features.

\section{Systems, Data, Methods}

The learning experiments that we describe here use the machine learning program RIPPER (Cohen, 1996) to automatically induce a "poor speech recognition performance" classification model from a corpus of spoken dialogues. ${ }^{1}$ RIPPER (like other learning programs, such as C5.0 and CART) takes as input the names of a set of classes to be learned, the names and possible values of a fixed set of features, training data specifying the class and feature values for each example in a training set, and outputs a classification model for predicting the class of future examples from their feature representation. In RIPPER, the classification model is learned using greedy search guided by an information gain metric, and is expressed as an ordered set of if-then rules. We use RIPPER for our experiments because it supports the use of "set-valued" features for representing text, and because if-then rules are often easier for people to understand than decision trees (Quinlan, 1993). Below we describe our corpus of dialogues, the assignment of classes to each dialogue, the extraction of features from each dialogue, and our learning experiments.

Corpus: Our corpus consists of a set of $544 \mathrm{di}$ alogues (over 40 hours of speech) between humans and one of three dialogue systems: ANNIE (Kamm et al., 1998), an agent for voice dialing and messaging; ELVIS (Walker et al., 1998b), an agent for accessing email; and TOOT (Litman and Pan, 1999), an agent for accessing online train schedules. Each agent was implemented using a generalpurpose platform for phone-based spoken dialogue systems (Kamm et al., 1997). The dialogues were obtained in controlled experiments designed to evaluate dialogue strategies for each agent. The exper-

\footnotetext{
${ }^{1}$ We also ran experiments using the machine learning program BoOSTEXTER (Schapire and Singer, To appear), with results similar to those presented below.
} 
iments required users to complete a set of application tasks in conversations with a particular version of the agent. The experiments resulted in both a digitized recording and an automatically produced system log for each dialogue.

Class Assignment: Our corpus is used to construct the machine learning classes as follows. First, each utterance that was not rejected by automatic speech recognition (ASR) was manually labeled as to whether it had been semantically misrecognized or not. ${ }^{2}$ This was done by listening to the recordings while examining the corresponding system log. If the recognizer's output did not correctly capture the task-related information in the utterance, it was labeled as a misrecognition. For example, in Figure $1 \mathrm{U} 4$ and U6 would be labeled as correct recognitions, while U5 and U7 would be labeled as misrecognitions. Note that our labeling is semantically based; if U5 had been recognized as "play messages" (which invokes the same application command as "get messages"), then U5 would have been labeled as a correct recognition. Although this labeling needs to be done manually, the labeling is based on objective criteria.

Next, each dialogue was assigned a class of either good or bad, by thresholding on the percentage of user utterances that were labeled as ASR semantic misrecognitions. We use a threshold of $11 \%$ to balance the classes in our corpus, yielding 283 good and 261 bad dialogues. ${ }^{3}$ Our classes thus reflect relative goodness with respect to a corpus. Dialogue D1 in Figure 1 would be classified as "bad", because U5 and U7 (29\% of the user utterances) are misrecognized.

Feature Extraction: Our corpus is used to construct the machine learning features as follows. Each dialogue is represented in terms of the 23 primitive features in Figure 2. In RIPPER, feature values are continuous (numeric), set-valued, or symbolic. Feature values were automatically computed from system logs, based on five types of knowledge sources: acoustic, dialogue efficiency, dialogue quality, experimental parameters, and lexical. Previous work correlating misrecognition rate with acoustic information, as well as our own

\footnotetext{
${ }^{2}$ These utterance labelings were produced during a previous set of experiments investigating the performance evaluation of spoken dialogue systems (Walker et al., 1997; Walker et al., 1998a; Walker et al., 1998b; Kamm et al., 1998; Litman et al., 1998; Litman and Pan, 1999).

${ }^{3}$ This threshold is consistent with a threshold inferred from human judgements (Litman, 1998).
}

- Acoustic Features

- mean confidence, pmisrecs $\% 1$, pmisrecs\%2, pmisrecs $\% 3$, pmisrecs $\% 4$

- Dialogue Efficiency Features

- elapsed time, system turns, user turns

- Dialogue Quality Features

- rejections, timeouts, helps, cancels, bargeins (raw)

- rejection\%, timeout\%, help\%, cancel\%, bargein\% (normalized)

- Experimental Parameters Features

- system, user, task, condition

- Lexical Features

- ASR text

Figure 2: Features for spoken dialogues.

hypotheses about the relevance of other types of knowledge, contributed to our features.

The acoustic, dialogue efficiency, and dialogue quality features are all numeric-valued. The acoustic features are computed from each utterance's confidence (log-likelihood) scores (Zeljkovic, 1996). Mean confidence represents the average log-likelihood score for utterances not rejected during ASR. The four pmisrecs\% (predicted percentage of misrecognitions) features represent different (coarse) approximations to the distribution of $\log$-likelihood scores in the dialogue. Each pmisrecs\% feature uses a fixed threshold value to predict whether a non-rejected utterance is actually a misrecognition, then computes the percentage of user utterances in the dialogue that correspond to these predicted misrecognitions. (Recall that our dialogue classifications were determined by thresholding on the percentage of actual misrecognitions.) For instance, pmisrecs $\% 1$ predicts that if a non-rejected utterance has a confidence score below -2 then it is a misrecognition. Thus in Figure 1, utterances U5 and $U 7$ would be predicted as misrecognitions using this threshold. The four thresholds used for the four pmisrecs\% features are $-2,-3,-4,-5$, and were chosen by hand from the entire dataset to be informative.

The dialogue efficiency features measure how quickly the dialogue is concluded, and include elapsed time (the dialogue length in seconds), and system turns and user turns (the number of turns for each dialogue participant). 


\begin{tabular}{|c|c|c|c|c|c|c|c|}
\hline $\begin{array}{l}\text { mean confidence } \\
-2.7\end{array}$ & $\begin{array}{l}\text { pmisrecs\%l } \\
29\end{array}$ & $\begin{array}{l}\text { pmisrecs } \% 2 \\
29\end{array}$ & $\begin{array}{l}\text { pmisrecs\%3 } \\
0\end{array}$ & $\begin{array}{l}\text { pmisrecs\%4 } \\
0\end{array}$ & $\begin{array}{l}\text { elapsed time } \\
300\end{array}$ & $\begin{array}{l}\text { system turns } \\
7\end{array}$ & $\begin{array}{l}\text { user turns } \\
7\end{array}$ \\
\hline rejections & timeouts & helps & cancels & bargeins & rejection\% & timeout\% & help\% \\
\hline 3 & 0 & 2 & 0 & 1 & 43 & 0 & 29 \\
\hline $\begin{array}{l}\text { cancel\% } \\
0\end{array}$ & $\begin{array}{l}\text { bargein\% } \\
14\end{array}$ & $\begin{array}{l}\text { system } \\
\text { annie }\end{array}$ & $\begin{array}{l}\text { user } \\
\text { mike }\end{array}$ & $\begin{array}{l}\text { task } \\
\text { day } 1\end{array}$ & $\begin{array}{l}\text { condition } \\
\text { novices without tutorial }\end{array}$ & & \\
\hline
\end{tabular}

Figure 3: Feature representation of dialogue D1.

The dialogue quality features attempt to capture aspects of the naturalness of the dialogue. Rejections represents the number of times that the system plays special rejection prompts, e.g., utterances $A 2$ and $A 3$ in dialogue D1. This occurs whenever the ASR confidence score falls below a threshold associated with the ASR grammar for each system state (where the threshold was chosen by the system designer). The rejections feature differs from the pmisrecs\% features in several ways. First, the pmisrecs $\%$ thresholds are used to determine misrecognitions rather than rejections. Second, the pmisrecs\% thresholds are fixed across all dialogues and are not dependent on system state. Third, a system rejection event directly influences the dialogue via the rejection prompt, while the pmisrecs\% thresholds have no corresponding behavior.

Timeouts represents the number of times that the system plays special timeout prompts because the user hasn't responded within a pre-specified time frame. Helps represents the number of times that the system responds to a user request with a (contextsensitive) help message. Cancels represents the number of user's requests to undo the system's previous action. Bargeins represents the number of user attempts to interrupt the system while it is speaking. ${ }^{4}$ In addition to raw counts, each feature is represented in normalized form by expressing the feature as a percentage. For example, rejection\% represents the number of rejected user utterances divided by the total number of user utterances.

In order to test the effect of having the maximum amount of possibly relevant information available, we also included a set of features describing the experimental parameters for each dialogue (even though we don't expect rules incorporating such features to generalize). These features capture the conditions under which each dialogue was col-

\footnotetext{
${ }^{4}$ Since the system automatically detects when a bargein occurs, this feature could have been automatically logged. However, because our system did not log bargeins, we had to handlabel them.
}

lected. The experimental parameters features each have a different set of user-defined symbolic values. For example, the value of the feature system is either "annie", "elvis", or "toot", and gives RIPPER the option of producing rules that are system-dependent.

The lexical feature ASR text is set-valued, and represents the transcript of the user's utterances as output by the ASR component.

Learning Experiments: The final input for learning is training data, i.e., a representation of a set of dialogues in terms of feature and class values. In order to induce classification rules from a variety of feature representations our training data is represented differently in different experiments. Our learning experiments can be roughly categorized as follows. First, examples are represented using all of the features in Figure 2 (to evaluate the optimal level of performance). Figure 3 shows how Dialogue D1 from Figure 1 is represented using all 23 features. Next, examples are represented using only the features in a single knowledge source (to comparatively evaluate the utility of each knowledge source for classification), as well as using features from two or more knowledge sources (to gain insight into the interactions between knowledge sources). Finally, examples are represented using feature sets corresponding to hypotheses in the literature (to empirically test theoretically motivated proposals).

The output of each machine learning experiment is a classification model learned from the training data. To evaluate these results, the error rates of the learned classification models are estimated using the resampling method of cross-validation (Weiss and Kulikowski, 1991). In 25-fold cross-validation, the total set of examples is randomly divided into 25 disjoint test sets, and 25 runs of the learning program are performed. Thus, each run uses the examples not in the test set for training and the remaining examples for testing. An estimated error rate is obtained by averaging the error rate on the testing portion of the data from each of the 25 runs. 


\begin{tabular}{|r|c|}
\hline Features Used & Accuracy (Standard Error) \\
\hline \hline BASELINE & $52 \%$ \\
\hline REJECTION\% & $54.5 \%(2.0)$ \\
\hline EFFICIENCY & $61.0 \%(2.2)$ \\
\hline EXP-PARAMS & $65.5 \%(2.2)$ \\
\hline DIALOGUE QUALITY (NORMALIZED) & $65.9 \%(1.9)$ \\
\hline MEAN CONFIDENCE & $68.4 \%(2.0)$ \\
\hline EFFICIENCY + NORMALIZED QUALITY & $69.7 \%(1.9)$ \\
\hline ASR TEXT & $72.0 \%(1.7)$ \\
\hline PMISRECS\%3 & $72.6 \%(2.0)$ \\
\hline EFFICIENCY + QUALITY + EXP-PARAMS & $73.4 \%(1.9)$ \\
\hline ALL FEATURES & $77.4 \%(2.2)$ \\
\hline
\end{tabular}

Figure 4: Accuracy rates for dialogue classifiers using different feature sets, 25-fold cross-validation on 544 dialogues. We use SMALL CAPS to indicate feature sets, and ITALICS to indicate primitive features listed in Figure 2.

\section{Results}

Figure 4 summarizes our most interesting experimental results. For each feature set, we report accuracy rates and standard errors resulting from crossvalidation..$^{5}$ It is clear that performance depends on the features that the classifier has available. The BASELINE accuracy rate results from simply choosing the majority class, which in this case means predicting that the dialogue is always "good". This leads to a 52\% BASELINE accuracy.

The REJECTION\% accuracy rates arise from a classifier that has access to the percentage of dialogue utterances in which the system played a rejection message to the user. Previous research suggests that this acoustic feature predicts misrecognitions because users modify their pronunciation in response to system rejection messages in such a way as to lead to further misunderstandings (Shriberg et al., 1992; Levow, 1998). However, despite our expectations, the REJECTION\% accuracy rate is not better than the BASELINE at our desired level of statistical significance.

Using the EFFICIENCY features does improve the performance of the classifier significantly above the BASELINE (61\%). These features, however, tend to reflect the particular experimental tasks that the users were doing.

The EXP-PARAMS (experimental parameters) features are even more specific to this dialogue corpus than the efficiency features: these features consist of the name of the system, the experimen-

\footnotetext{
${ }^{5}$ Accuracy rates are statistically significantly different when the accuracies plus or minus twice the standard error do not overlap (Cohen, 1995), p. 134.
}

tal subject, the experimental task, and the experimental condition (dialogue strategy or user expertise). This information alone allows the classifier to substantially improve over the BASELINE classifier, by identifying particular experimental conditions (mixed initiative dialogue strategy, or novice users without tutorial) or systems that were run with particularly hard tasks (TOOT) with bad dialogues, as in Figure 5. Since with the exception of the experimental condition these features are specific to this corpus, we wouldn't expect them to generalize.

if $($ condition $=$ mixed $)$ then $b a d$

if (system $=$ toot) then bad

if (condition $=$ novices without tutorial) then $b a d$

default is good

Figure 5: EXP-PARAMS rules.

The normalized DIALOGUE QUALITY features result in a similar improvement in performance $(65.9 \%){ }^{6}$ However, unlike the efficiency and experimental parameters features, the normalization of the dialogue quality features by dialogue length means that rules learned on the basis of these features are more likely to generalize.

Adding the efficiency and normalized quality feature sets together (EFFICIENCY + NORMALIZED QUALITY) results in a significant performance improvement $(69.7 \%)$ over EFFICIENCY alone. Figure 6 shows that this results in a classifier with three rules: one based on quality alone (percentage of cancellations), one based on efficiency

\footnotetext{
${ }^{6}$ The normalized versions of the quality features did better than the raw versions.
} 
alone (elapsed time), and one that consists of a boolean combination of efficiency and quality features (elapsed time and percentage of rejections). The learned ruleset says that if the percentage of cancellations is greater than $6 \%$, classify the dialogue as bad; if the elapsed time is greater than 282 seconds, and the percentage of rejections is greater than $6 \%$, classify it as bad; if the elapsed time is less than 90 seconds, classify it as $\mathrm{bad}^{7}$; otherwise classify it as good. When multiple rules are applicable, RIPPER resolves any potential conflict by using the class that comes first in the ordering; when no rules are applicable, the default is used.

if (cancel\% $>6$ ) then bad

if (elapsed time $\geq 282$ secs) $\wedge$ (rejection\% $\geq 6$ ) then bad

if (elapsed time $<90$ secs) then bad

default is good

Figure 6: EFFICIENCY + NORMALIZED QUALITY rules.

We discussed our acoustic REJECTION\% results above, based on using the rejection thresholds that each system was actually run with. However, a posthoc analysis of our experimental data showed that our systems could have rejected substantially more misrecognitions with a rejection threshold that was lower than the thresholds picked by the system designers. (Of course, changing the thresholds in this way would have also increased the number of rejections of correct ASR outputs.) Recall that the PMISRECS\% experiments explored the use of different thresholds to predict misrecognitions. The best of these acoustic thresholds was PMISRECS\%3, with accuracy $72.6 \%$. This classifier learned that if the predicted percentage of misrecognitions using the threshold for that feature was greater than $8 \%$, then the dialogue was predicted to be bad, otherwise it was good. This classifier performs significantly better than the BASELINE, $R E$ $J E C T I O N \%$ and EFFICIENCY classifiers.

Similarly, MEAN CONFIDENCE is another acoustic feature, which averages confidence scores over all the non-rejected utterances in a dialogue. Since this feature is not tuned to the applications, we did not expect it to perform as well as the best PMISRECS\% feature. However, the accuracy rate

\footnotetext{
${ }^{7}$ This rule indicates dialogues too short for the user to have completed the task. Note that this rule could not be applied to adapting the system's behavior during the course of the dialogue.
}

for the MEAN CONFIDENCE classifier (68.4\%) is not statistically different than that for the PMIS$R E C S \% 3$ classifier. Furthermore, since the feature does not rely on picking an optimal threshold, it could be expected to better generalize to new dialogue situations.

The classifier trained on (noisy) ASR lexical output (ASR TEXT) has access only to the speech recognizer's interpretation of the user's utterances. The ASR TEXT classifier achieves $72 \%$ accuracy, which is significantly better than the BASELINE, REJECTION\% and EFFICIENCY classifiers. Figure 7 shows the rules learned from the lexical feature alone. The rules include lexical items that clearly indicate that a user is having trouble e.g. help and cancel. They also include lexical items that identify particular tasks for particular systems, e.g. the lexical item $p$ - $m$ identifies a task in TOOT.

if (ASR text contains cancel) then bad

if (ASR text contains the) $\wedge$ (ASR text contains get) $\wedge$ (ASR text contains T IMEOUT) then bad

if (ASR text contains today) $\wedge$ (ASR text contains on) then bad if (ASR text contains the) $\wedge$ (ASR text contains $p-m$ ) then bad if (ASR text contains to) then bad

if $($ ASR text contains help) $\wedge$ (ASR text contains the) $\wedge$ (ASR text contains read) then bad

if (ASR text contains help) $\wedge$ (ASR text contains previous) then bad

if (ASR text contains about) then bad

if (ASR text contains change-strategy) then bad

default is good

Figure 7: ASR TEXT rules.

Note that the performance of many of the classifiers is statistically indistinguishable, e.g. the performance of the ASR TEXT classifier is virtually identical to the classifier PMISRECS\% 3 and the EFFICIENCY + QUALITY + EXP-PARAMS classifier. The similarity between the accuracies for a range of classifiers suggests that the information provided by different feature sets is redundant. As discussed above, each system and experimental condition resulted in dialogues that contained lexical items that were unique to it, making it possible to identify experimental conditions from the lexical items alone. Figure 8 shows the rules that RIPPER learned when it had access to all the features except for the lexical and acoustic features. In this case, RIPPER learns some rules that are specific to the TOOT system.

Finally, the last row of Figure 4 suggests that a classifier that has access to ALL FEATURES may do better ( $77.4 \%$ accuracy) than those classifiers that 
if $($ cance $1 \%>4) \wedge$ (system $=$ toot) then bad

if (system turns $\geq 26) \wedge$ (rejection $\% \geq 5$ ) then $b a d$

if (condition $=$ mixed) $\wedge$ (user turns $\geq 12$ ) then bad

if (system $=$ toot) $\wedge$ (user turns $\geq 14$ ) then bad

if (cancels $\geq 1) \wedge$ (timeout\% $\geq 11$ ) then bad

if (elapsed time $\leq 87$ secs) then bad

default is good

Figure 8: EFFICIENCY + QUALITY + EXP-PARAMS rules.

have access to acoustic features only $(72.6 \%)$ or to lexical features only $(72 \%)$. Although these differences are not statistically significant, they show a trend $(\mathrm{p}<.08)$. This supports the conclusion that different feature sets provide redundant information, and could be substituted for each other to achieve the same performance. However, the ALL FEATURES classifier does perform significantly better than the EXP-PARAMS, DIALOGUE QUALITY (NORMALIZED), and MEAN CONFIDENCE classifiers. Figure 9 shows the decision rules that the ALL FEATURES classifier learns. Interestingly, this classifier does not find the features based on experimental parameters to be good predictors when it has other features to choose from. Rather it combines features representing acoustic, efficiency, dialogue quality and lexical information.

if (mean confidence $\leq-2.2) \wedge($ pmisrecs $\% 4 \geq 6)$ then bad if (pmisrecs\% $3 \geq 7$ ) $\wedge$ (ASR text contains yes) $\wedge$ (mean confidence $\leq-1.9)$ then $b a \bar{d}$

if (cancel\% $\geq 4$ ) then bad

if (system turns $\geq 29) \wedge$ (ASR text contains message) then bad

if (elapsed time $\leq 90$ ) then bad

default is good

Figure 9: ALL FEATURES rules.

\section{Discussion}

The experiments presented here establish several findings. First, it is possible to give an objective definition for poor speech recognition at the dialogue level, and to apply machine learning to build classifiers detecting poor recognition solely from features of the system log. Second, with appropriate sets of features, these classifiers significantly outperform the baseline percentage of the majority class. Third, the comparable performance of classifiers constructed from rather different feature sets (such as acoustic and lexical features) suggest that there is some redundancy between these feature sets (at least with respect to the task). Fourth, the fact that the best estimated accuracy was achieved using all of the features suggests that even problems that seem inherently acoustic may best be solved by exploiting higher-level information.

This work differs from previous work in focusing on behavior at the (sub)dialogue level, rather than on identifying single misrecognitions at the utterance level (Smith, 1998; Levow, 1998; van Zanten, 1998). The rationale is that a single misrecognition may not warrant a global change in dialogue strategy, whereas a user's repeated problems communicating with the system might warrant such a change. While we are not aware of any other work that has applied machine learning to detecting patterns suggesting that the user is having problems over the course of a dialogue, (Levow, 1998) has applied machine learning to identifying single misrecognitions. We are currently extending our feature set to include acoustic-prosodic features such as those used by Levow, in order to predict misrecognitions at both the dialogue level as well as the utterance level.

We are also interested in the extension and generalization of our findings in a number of additional directions. In other experiments, we demonstrated the utility of allowing the user to dynamically adapt the system's dialogue strategy at any point(s) during a dialogue. Our results show that dynamic adaptation clearly improves system performance, with the level of improvement sometimes a function of the system's initial dialogue strategy (Litman and Pan, 1999). Our next step is to incorporate classifiers such as those presented in this paper into a system in order to support dynamic adaptation according to recognition performance. Another area for future work would be to explore the utility of using alternative methods for classifying dialogues as good or bad. For example, the user satisfaction measures we collected in a series of experiments using the PARADISE evaluation framework (Walker et al., 1998c) could serve as the basis for such an alternative classification scheme. More generally, in the same way that learning methods have found widespread use in speech processing and other fields where large corpora are available, we believe that the construction and analysis of spoken dialogue systems is a ripe domain for machine learning applications.

\section{Acknowledgements}

Thanks to J. Chu-Carroll, W. Cohen, C. Kamm, M. Kan, R. Schapire, Y. Singer, B. Srinivas, and S. 
Whittaker for help with this research and/or paper.

\section{References}

Paul R. Cohen. 1995. Empirical Methods for Artificial Intelligence. MIT Press, Boston.

William Cohen. 1996. Learning trees and rules with set-valued features. In 14th Conference of the American Association of Artificial Intelligence, AAAI.

C. Kamm, S. Narayanan, D. Dutton, and R. Ritenour. 1997. Evaluating spoken dialog systems for telecommunication services. In 5th European Conference on Speech Technology and Communication, EUROSPEECH 97.

Candace Kamm, Diane Litman, and Marilyn A. Walker. 1998. From novice to expert: The effect of tutorials on user expertise with spoken dialogue systems. In Proceedings of the International Conference on Spoken Language Processing, ICSLP98.

Gina-Anne Levow. 1998. Characterizing and recognizing spoken corrections in human-computer dialogue. In Proceedings of the 36th Annual Meeting of the Association of Computational Linguistics, COLING/ACL 98, pages 736-742.

Diane J. Litman and Shimei Pan. 1999. Empirically evaluating an adaptable spoken dialogue system. In Proceedings of the 7th International Conference on User Modeling (UM).

Diane J. Litman, Shimei Pan, and Marilyn A. Walker. 1998. Evaluating Response Strategies in a Web-Based Spoken Dialogue Agent. In Proceedings of ACL/COLING 98: 36th Annual Meeting of the Association of Computational Linguistics, pages 780-787.

Diane J. Litman. 1998. Predicting speech recognition performance from dialogue phenomena. Presented at the American Association for Artificial Intelligence Spring Symposium Series on Applying Machine Learning to Discourse Processing.

J. Ross Quinlan. 1993. C4.5: Programs for $M a-$ chine Learning. San Mateo, CA: Morgan Kaufmann.

Robert E. Schapire and Yoram Singer. To appear. Boostexter: A boosting-based system for text categorization. Machine Learning.

Elizabeth Shriberg, Elizabeth Wade, and Patti Price. 1992. Human-machine problem solving using spoken language systems (SLS): Factors affecting performance and user satisfaction. In Pro- ceedings of the DARPA Speech and NL Workshop, pages 49-54.

Ronnie W. Smith. 1998. An evaluation of strategies for selectively verifying utterance meanings in spoken natural language dialog. International Journal of Human-Computer Studies, 48:627647.

G. Veldhuijzen van Zanten. 1998. Adaptive mixedinitiative dialogue management. Technical Report 52, IPO, Center for Research on UserSystem Interaction.

Marilyn Walker, Donald Hindle, Jeanne Fromer, Giuseppe Di Fabbrizio, and Craig Mestel. 1997. Evaluating competing agent strategies for a voice email agent. In Proceedings of the European Conference on Speech Communication and Technology, EUROSPEECH97.

M. Walker, J. Fromer, G. Di Fabbrizio, C. Mestel, and D. Hindle. 1998a. What can I say: Evaluating a spoken language interface to email. In Proceedings of the Conference on Computer Human Interaction (CHI 98).

Marilyn A. Walker, Jeanne C. Fromer, and Shrikanth Narayanan. 1998b. Learning optimal dialogue strategies: A case study of a spoken dialogue agent for email. In Proceedings of the 36th Annual Meeting of the Association of Computational Linguistics, COLING/ACL 98, pages 1345-1352.

Marilyn. A. Walker, Diane J. Litman, Candace. A. Kamm, and Alicia Abella. 1998c. Evaluating spoken dialogue agents with PARADISE: Two case studies. Computer Speech and Language, 12(3).

S. M. Weiss and C. Kulikowski. 1991. Computer Systems That Learn: Classification and Prediction Methods from Statistics, Neural Nets, Machine Learning, and Expert Systems. San Mateo, CA: Morgan Kaufmann.

Ilija Zeljkovic. 1996. Decoding optimal state sequences with smooth state likelihoods. In International Conference on Acoustics, Speech, and Signal Processing, ICASSP 96, pages 129-132. 